\title{
ANÁLISIS DE LA RECOMENDACIÓN ENTRE IGUALES EN LA REPUTACIÓN ONLINE DE LAS ORGANIZACIONES
}

\author{
Analysis of peer recommendations in the online \\ reputation of organizations
}

\section{Lucía Benítez-Eyzaguirre}

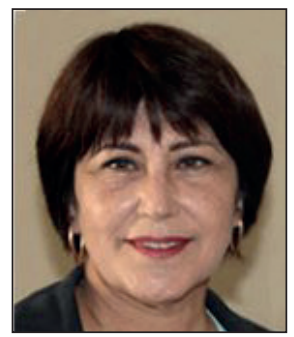

Lucía Benítez-Eyzaguirre, profesora de la Universidad de Cádiz, es doctora por la Universidad de Sevilla (con premio extraordinario) y premio RTVA a la mejor tesis doctoral de comunicación. Master en tecnologías digitales y sociedad del conocimiento. Directora de la revista científica Redes. com. Miembro del grupo de investigación Compolíticas, del campo de la comunicación para el desarrollo. Participa en proyectos de investigación y de enseñanza del periodismo y las nuevas tecnologías en Marruecos, Guinea Ecuatorial y Cabo Verde.

http://orcid.org/0000-0002-8879-7979

Universidad de Cádiz, Departamento de Marketing y Comunicación Campus Universitario de Jerez, Avda. de la Universidad. 11405 Jerez de la Frontera (Cádiz), España lucia.benitez@gm.uca.es

\section{Resumen}

La reputación corporativa se ve afectada por el creciente impacto de la reputación online, especialmente por el peso que adquiere la recomendación entre iguales en los medios sociales. El prosumidor (McLuhan; Nevitt, 1972; Toffler, 1981; Tapscott, 1997) tiene una autonomía y capacidad en la Red que le hace incorporarse a los agentes implicados como un emergente surgido del contexto digital, escasamente teorizado en relación con la reputación corporativa. En el contexto de la definición de estos conceptos, este artículo propone modelos de análisis de la reputación online en función de las acciones comunicativas de los prosumidores, desde un enfoque cualitativo para abordar el conocimiento de los públicos y de los intervinientes. Para ello, realiza una revisión teórica crítica fundamentada sobre fuentes secundarias.

\section{Palabras clave}

Prosumidor; Reputación online; Comunicación organizacional; Recomendación entre iguales.

\section{Abstract}

Corporate reputation is affected by the growing impact of online reputation, because of the weight of peer recommendations in social media. Digital prosumers' (McLuhan; Nevitt, 1972; Toffler, 1981; Tapscott, 1997) autonomy and ability on the internet have led them to integrate with stakeholders. Their role in relation to corporate reputation has barely been recognized. In the context of the definition of these concepts, this article proposes analysis models and tools for online reputation study, based on the communicative actions of prosumers. We take a qualitative methodology as the basic strategy to approach the knowledge of the public and the stakeholders. We carry out a critical theoretical review based on secondary sources.

\section{Keywords}

Prosumer; Online reputation; Organizational communication; Peer recommendation.

Benítez-Eyzaguirre, Lucía (2016). “Análisis de la recomendación entre iguales en la reputación online de las organizaciones". El profesional de la información, v. 25, n. 4, pp. 652-660.

http://dx.doi.org/10.3145/epi.2016.jul.15

\section{Introducción}

Las transformaciones tecnológicas sitúan a las organizaciones y sus públicos en un plano de igualdad en sus intercambios comunicativos, en función del ecosistema horizontal propiciado por internet. Si antes las organizaciones podían desempeñar el monopolio y la dirección de sus acciones de comunicación, en el actual contexto la estructura iguala la emisión y recepción de mensajes, que ya no son difusionistas sino conversacionales.

Los usuarios son prosumidores, es decir, productores y con- 
sumidores de contenidos con capacidad y poder de alterar la reputación de las organizaciones. Sus acciones y conversaciones se desarrollan al margen de las empresas, en un sistema abierto y democratizado de difícil control y gestión para la reputación corporativa, por cuanto la interacción entre iguales (Tapscott; Williams, 2007) y la reputación colectiva operan desde abajo.

Se propone aquí una metodología de análisis de la conversación de los prosumidores como parte de la gestión de la reputación online.

Los usuarios son prosumidores, es decir, productores y consumidores de contenidos con capacidad y poder de alterar la reputación de las organizaciones

\section{Metodología y objetivos}

La reputación online forma parte esencial de la imagen de la empresa, aunque en su definición hay diferentes perspectivas. Justo Villafañe no diferencia el contexto digital en la construcción de la reputación, es decir, defiende el concepto como único por encima del canal en que se registre: "No existe una reputación online y una offline, la reputación es una".

Sin embargo, Hernández (2011) entiende que es complementario, ya que el circuito de la comunicación digital se suma al que se registra offline: "La reputación online es el reconocimiento que los stakeholders (o personas implicadas o intervinientes) de una compañía manifiestan en internet sobre el comportamiento corporativo de la misma a partir del grado de cumplimiento de sus compromisos". En la práctica, muchos profesionales entienden la reputación online a partir del análisis y la recopilación de datos sobre las opiniones en internet y que, por tanto, sólo es necesario atender a la monitorización. Este enfoque olvida la transformación fundamental del papel de los públicos a partir de la figura del prosumidor, que avanzó Tapscott (1997), y que describía su actividad y su perfil como una figura híbrida entre la producción y el consumo de contenidos, bajo la filosofía de la autonomía y la colaboración. En este sentido se puede entender a los prosumidores como stakeholders de las corporaciones y protagonistas activos de la comunicación online.

El concepto de reputación online, en función de los cambios registrados a partir de la web 2.0, precisa una reformulación porque los usuarios registran una conversación multidireccional y compleja que resulta de difícil análisis. Este comportamiento deja obsoleto el concepto de la reputación online basada en la actividad SEO (search engine optimization) e incluso el que entiende que la cuestión está relacionada con la monitorización de redes sociales o con la gestión de crisis. Se trata de mucho más, a la vista del papel protagonista que la interacción entre iguales, la valoración y opiniones en redes sociales, la autonomía y poder de los prosumidores en la red. Partiendo de la idea de que la reputación online forma parte de la comunicación corporativa, se plantea aquí la necesidad de mejorar la investigación y el análisis de los comportamientos y prácticas de los usuarios y prosumidores.
Este artículo parte de la hipótesis de que en el contexto de la conversación online la reputación se construye desde abajo con la participación de los prosumidores como usuarios muy activos que contribuyen a la reputación colectiva, a través de la recomendación entre iguales (Williamson; Stayner 1980), un modelo de producción de confianza a partir de la valoración de los públicos. El poder de los usuarios en la construcción del valor de las compañías se debe a su autonomía comunicativa y a su doble condición de productores y consumidores, que obliga a entender a los públicos como parte de los stakeholders en la estrategia de las compañías.

Con esta hipótesis, el objetivo de la presente investigación es establecer criterios de análisis sobre la recomendación entre iguales como parte de la comunicación corporativa, así como proponer metodologías cualitativas, más allá de la monitorización cuantitativa de las redes. La recomendación entre iguales (Williamson; Stayner 1980; Light, 2001) se entiende como un modelo de confianza generado por los clientes y usuarios de bienes y servicios a partir de la interacción online. Por tanto, este intercambio de opiniones tiene impacto sobre la reputación de las empresas.

A través del análisis fundamentado sobre fuentes secundarias, de un arco teórico y contextual fruto del análisis documental, se busca una redefinición de los públicos en función de su papel de prosumidores en la construcción de la reputación corporativa. Se describe la evolución del contexto digital para la nueva concepción de la actividad empresarial, de cara a las transformaciones económicas así como a su impacto sobre la gestión del management. Se profundiza en el objeto de estudio con la propuesta de modelos de estudio de la recomendación entre iguales, de la participación de los prosumidores en la conversación en red y de la influencia que ejercen sobre la reputación online.

En el contexto de la conversación online, la reputación se construye desde abajo con la participación de los prosumidores como usuarios activos que contribuyen a la reputación colectiva

\section{La reputación online en las relaciones públicas}

Con la web 2.0 y la aparición del prosumidor como figura híbrida en el contexto de la convergencia multicanal de internet, se registra una nueva fuente de creación de valor en la conversación global. McLuhan y Nevitt (1972) describieron por primera vez y con carácter prescriptor el comportamiento de este tipo de usuario. Toffler (1981) anticipó después el fin de los grandes medios masivos y de los modelos de control en la comunicación, como un cambio irreversible en el que los prosumidores tienen capacidad para incidir y transformar los mercados.

La acción conjunta de los públicos invierte el modelo de mando desarrollado en la gestión comunicativa, ya que se entiende que la reputación se impulsa también desde abajo por el efecto de la participación de los usuarios en la conversación online. En el campo de la comunicación, 
las redes sociales han ocasionado la ruptura del tradicional paradigma de la comunicación de Lasswell para incorporar una estructura adecuada a la convergencia y la multidireccionalidad horizontal, con integración de los públicos en tanto que prosumidores y nuevos stakeholders en las corporaciones.

La reputación online forma parte en sí misma de la reputación corporativa, por lo que debe entenderse y valorarse según los mismos criterios. Dada la importancia creciente de la recomendación entre iguales en la reputación online y, por tanto, corporativa, se trata aquí de presentar criterios de evaluación y análisis de la actividad de los prosumidores a partir de las siguientes cuestiones esenciales:

3.1. La digitalización ha supuesto cambios económicos y sociales definitivos en la relación comunicativa con los stakeholders y los públicos, que debe ser contemplada de cara a la conceptualización de la reputación online.

3.2. El papel de la recomendación entre iguales dentro de la reputación online y corporativa, en el nuevo ecosistema comunicativo, tiene un peso decisivo por el efecto de las acciones y la experiencia de los prosumidores sobre la reputación de las compañías.

3.3. La evaluación de la reputación corporativa precisa de la inclusión de la conversación y la recomendación entre iguales por el valor que tienen para la estrategia corporativa, a través de la participación y la colaboración de los prosumidores.

3.4. Los modelos de análisis de la reputación online deben contemplar valores cualitativos para describir las acciones de los usuarios ya que son una fuente de conocimiento esencial para la gestión de la reputación social de las empresas.

3.5. La reputación corporativa suma los cauces online y offline porque se funden en una única experiencia, que se realimenta de las acciones y las prácticas de los públicos. De la misma forma que la convergencia propuesta en los procesos de digitalización, la integración de la comunicación digital y presencial dentro de una misma estrategia es la forma coherente de analizar estos procesos.

La recomendación entre iguales se entiende como un modelo de confianza generado por los clientes y usuarios de bienes y servicios a partir de la interacción online

Por tanto, a partir de estas cuestiones pasamos a profundizar en las transformaciones económicas y sociales con impacto en la gestión corporativa.

\section{Empresas y organizaciones en el contexto digital}

La web 2.0 dibuja un escenario horizontal, participativo y más igualitario. Las transformaciones que describió Himanen (2002) sobre el espíritu de la sociedad de la información proporcionan las claves para el ejercicio del liderazgo y de la gestión empresarial, ya que la lógica colaborativa y la participación en la toma de decisiones facilitan la competitividad colectiva.

De una forma más concreta, y aplicado al contexto empresarial, Tapscott y Williams (2007) habían descrito el impacto económico de la inteligencia colectiva en su libro Wikinomics, a partir de las experiencias de colaboración, producción, y la influencia en el valor de los productos y servicios. Los blogs, redes sociales y otras plataformas activan una conversación permanente que afecta a la reputación de las organizaciones. Tapscott y Williams (2007) también definieron la 'interacción entre iguales' desde la paridad en el acceso a la información y la autoorganización. El modelo ha conducido a la creación colectiva de soluciones y productos que van desde la elaboración de software libre hasta la Wikipedia, todo un mundo de opciones desde la acción colectiva.

\section{La reputación corporativa suma los cau- ces online y offline porque se funden en una única experiencia, que se realimen- ta de las acciones y las prácticas de los públicos}

La wikinomía describe la estructura social y productiva de intercambios y de colaboración sin mando, a partir de las conexiones entre el interior de las organizaciones y las redes externas, en una estructura que permite otros modos de interacción, de producción y de trabajo, según los principios de la economía digital (apertura de los sistemas, interacción entre iguales, compartir y colaborar, y capacidad de actuación global). Todas ellas muestran tendencias opuestas a las que se han venido defendiendo dentro de la economía física y mercantil durante las décadas anteriores a la irrupción de internet. En estos principios se basan los modelos de colaboración masiva como nuevos prototipos productivos pero también son modelos de influencia para la reputación corporativa.

El Manifiesto Cluetrain (1999) propuso cambios en las empresas y organizaciones para responder a las nuevas prácticas de comunicación que influyen en los mercados. También en el caso español, el Manifiesto Crowd (Gutiérrez-Rubí; Freire, 2013) concreta los elementos esenciales en la transformación de la cultura corporativa con el impulso de la comunicación, de otros modos de organización y del reconocimiento del talento o de la meritocracia. Estos elementos coinciden con modos de relación de un sistema abierto, con otra forma de implementar productos y servicios, y establecen dinámicas a considerar en la gestión de la comunicación y de la reputación corporativa.

Estamos por tanto ante una nueva cultura empresarial y económica, en la que la conectividad obliga a rediseñar las estrategias de la comunicación corporativa y a cuestionar las métricas dominantes del análisis del comportamiento de los públicos. Este escenario acaba con las jerarquías y con el control de la comunicación, así como con la estructura vertical desde la que a menudo se ha gestionado la reputación corporativa. 


\section{La web 2.0 rompe el equilibrio de la comunicación corporativa}

O’Reilly (2005) definió la web 2.0 a partir de un software que ya no precisa de actualizaciones ni va unido a un dispositivo único porque es una plataforma en la que las experiencias de los usuarios perfeccionan los servicios ofertados. La inteligencia colectiva (Lévy, 2004) como concepto de la cultura digital hace referencia al concurso plural y la colaboración para la toma de decisiones, la explotación de la información de internet, la comparación y el estudio de los comportamientos colectivos, para la predicción y aprovechamiento de grandes datos en la toma de decisiones. Por tanto, la irrupción de la web 2.0 supuso cambios estructurales y tecnológicos como un ecosistema comunicativo y de acciones que ahora se caracteriza por la ausencia de mando. La estructura de la comunicación digital ha impuesto un proceso de desintermediación en las actividades empresariales, comerciales, sociales, económicas y políticas. Se rompe la cadena tradicional entre el emisor y el receptor, la relación entre los productores de contenidos y los receptores. Con el fin de la intermediación acaba el papel protagónico de los medios de comunicación y surge otro orden comunicativo de relaciones sociales y colectivas.

La conectividad obliga a rediseñar las estrategias de la comunicación corporativa y a cuestionar las métricas dominantes del análisis del comportamiento de los públicos

A partir de la web 2.0 también los buscadores pierden su papel protagonista en el acceso a contenidos para dar paso a las redes sociales. Si durante el primer desarrollo de la web el posicionamiento en buscadores o SEO (search engine optimization) ha sido esencial para mejorar la visibilidad y la notoriedad de las corporaciones dentro de internet, ahora la situación es otra, sobre todo si se trata de estudiar la reputación, que está más relacionada con el cumplimiento de acciones y compromisos.

El Manifiesto Cluetrain defiende la apertura y la horizontalidad de las empresas para adaptarse a la estructura que ha impuesto la red, en la que las organizaciones dependen de los vínculos para obtener como ventaja competitiva la inteligencia colectiva. El principio de apertura (Tapscott; Williams, 2007) exige cambios en el concepto de secreto empresarial, pues se entiende que el intercambio de información y la transparencia reducen los costes empresariales $y$, a la vez, proyectan una imagen de confianza. Por ello, el acceso a la información corporativa y el fin de los secretos forman parte de la filosofía de la cultura digital, y apuntan a otras tendencias en la recepción de la publicidad y en la gestión de las relaciones públicas, pues los usuarios con habilidades y competencias para obtener información directa exigen transparencia.

El modelo social y económico resultante lleva a considerar a los mercados como conversaciones, en las que las empresas se comunican de forma directa y abierta dentro de un sistema que se enriquece y gana valor añadido a partir de la experiencia (Levine et al., 1999). De cara a la comunicación, los autores recomiendan pautas diferenciales de comportamiento de las organizaciones, como sería la construcción de una masa crítica para recoger los beneficios que, a largo plazo, se producen. De hecho, el Manifiesto Cluetrain incorpora también la acción de los públicos como prosumidores, es decir, como parte del mercado y de la producción. En coherencia con la teoría de las relaciones públicas, se entiende que el fin último del proceso de comunicación y de alianzas está en establecer un diálogo entre organizaciones y públicos.

La web 2.0 dibuja un escenario horizontal, participativo y más igualitario

\section{La comunicación horizontal y el prosumidor como mensaje en la reputación online}

Las prácticas comunicativas de los prosumidores se desarrollan en un "espacio de encuentro y transformación en donde se articulan las percepciones plurales de la realidad" (Pérez; Massoni, 2009, p. 300). Esta visión compartida es definitiva en la comunicación y, aunque forma parte del capital reputacional de las empresas, se gestiona, cada vez más, al margen de estas instituciones y de las jerarquías (Tapscott; Williams, 2007, p. 80).

La interacción entre iguales configura comunidades colaborativas y autónomas -a menudo alrededor de gustos y preferencias- que vienen mostrando su eficacia en la gestión del conocimiento, pero también orientando consumos culturales como la lectura, la música o el cine. El prosumidor participa en los cambios culturales y transforma la relación entre producción y consumo, y los valores de marca (Toffler, 1981, p. 269), y con ello la reputación de las empresas y de sus productos, aun cuando no los consuma. En conjunto, las opiniones y valores de los prosumidores conducirían a la recomendación colectiva (crowdcuration), un concepto que surge del comercio electrónico como mecanismo de producción de confianza sobre las decisiones de compra en el que se apoyan muchos modelos de negocio como eBay o TripAdvisor.

La web social ha popularizado estos modelos de producción de confianza a partir de algoritmos que describen la información y las prácticas de los usuarios, que analizan sus preferencias y filtran la información, las opiniones y características de sus elecciones para orientar a los iguales. La monitorización del entorno y el valor de la inteligencia colectiva tienen capacidad para alterar y producir valor sobre los contenidos y la reputación online. Se entiende que la inteligencia colectiva puede superar el posible sesgo de los expertos a la hora de analizar la información de toda una organización, de cara a comprender comportamientos colectivos o a realizar predicciones. De hecho, la extracción de datos masivos o big data es una fuente de información relevante para las organizaciones.

Cabe aquí contemplar la capacidad de los usuarios para la transformación de los valores de marca de las empresas, que obliga a prestar atención a su impacto sobre la reputa- 
ción online, como un desarrollo innovador de la digitalización. El modelo de la recomendación entre iguales resulta más solvente conforme los prescriptores sean conocidos o estén valorados en la red, o bien por el valor colectivo de la recomendación social. El concepto, en el ámbito de las relaciones públicas y la comunicación, procede del clásico boca-oído y en el mundo digital se conoce como eWoM (electronic word of mouth) (Westbrook, 1987), que contempla la comunicación informal entre consumidores sobre las empresas, sus servicios y productos. EI valor de estas prescripciones puede ser superior al de los medios de comunicación ya que, además de estar libre de intereses comerciales, se autorregulan de forma abierta y transparente. En este sentido, forma parte de la tendencia de colaboración que mejora los servicios de las plataformas online (San-Millán-Fernández; Medrano-García; Cavarkapa, 2009, p. 4).

Con sus prácticas de comunicación, los usuarios terminan tejiendo una red, cuya acción colectiva y opiniones son la expresión de su poder en el mercado y, por tanto, de su impacto económico. Los prosumidores se erigen en jueces del desempeño organizacional, de la coherencia entre las acciones y la comunicación de las corporaciones, son testigos del compromiso empresarial con el entorno. Las experiencias y la valoración de los productos y bienes producen el entramado social de la reputación online que condiciona las percepciones de los públicos.

El peso del fenómeno, a la vista de las prácticas y de la acción colectiva, y del creciente número de plataformas que explotan la recomendación entre iguales, debe conducir a un cambio en la orientación de la estrategia de comunicación porque, por encima del efecto y la influencia que la gestión profesional pueda ejercer sobre los mensajes, la de la multitud inteligente logra mayor credibilidad y confianza en la reputación.

\section{El intercambio de información y la trans- parencia reducen los costes empresaria- les $y$, a la vez, proyectan una imagen de confianza}

\section{Estrategia de una reputación online}

En la web social, la interactividad propone un diálogo y un intercambio de opiniones en los que la escucha es también parte del proceso. Las organizaciones se deben insertar en el contexto social con apertura y flexibilidad para aprovechar las aportaciones de la inteligencia colectiva, de la innovación y creatividad. Así se entiende que, de cara a las relaciones públicas, más allá que publicidad y estrategia, el enfoque ha de ser el de la creación de una comunidad. En la construcción de la reputación online, son clave la participación en la conversación, los contenidos con acceso y enlaces, el análisis de los mensajes, la respuesta a los problemas y el estudio de la satisfacción. Los valores de la comunicación corporativa se transforman de acuerdo con el ecosistema digital en el que la cooperación es la base de la creación de alianzas.

\section{Manifiesto Crowd}

\section{La empresa y la inteligencia de las multitudes}

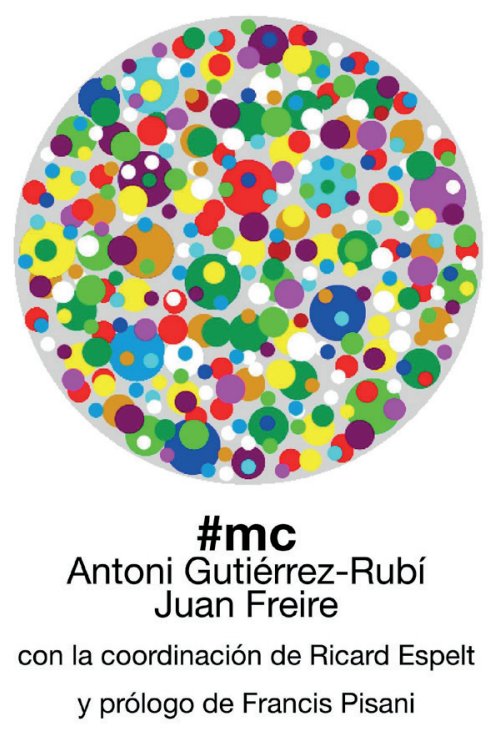

http://www.gutierrez-rubi.es/2013/03/18/manifiesto-crowd-libro-y-manifiesto

El prosumidor avala la recomendación de productos y servicios con voz propia, autónoma e independiente. Se transforma en influenciador en la medida en que su criterio sea independiente, y fruto de su capacidad de obtener información en la Red, de su propio conocimiento o de la valía de sus opiniones. En el mundo 2.0, la influencia de los usuarios, la posibilidad de convertirse o ejercer de líder es muy superior a la del mundo físico.

En su origen, se detectó esta tendencia especialmente para el caso de los consumos culturales, como lecturas o cine, donde son decisivos factores estéticos, intelectuales o emocionales (Stebbins, 1996, p. 948). El mensaje emocional es muy influyente y debe ser considerado en la gestión de la comunicación online, más todavía si tiene clave empática y si se enfoca hacia la mejora de la reputación, tal y como propone Cleminson (2012, p. 119):

"La empatía y su compañía, la emoción, son reclamadas para mejorar nuestros sentimientos hacia instituciones y entidades impersonales que están dañadas y sufren como el mercado".

Esta emoción social está en el origen de las visiones colectivas y de las relaciones. Por ello, el Manifiesto Crowd recomienda las relaciones emocionales con los stakeholders o participantes involucrados. En las percepciones se transforma el conocimiento en un sistema de necesidades que determina la acción, pero también por la relación emocional con el proceso y el objeto (Alloza, 2010, p. 134). La influencia social produce comportamientos imitativos, en los que se adoptan formas y expresiones similares a las que han conocido de otros iguales (Bonchi, 2011). De alguna forma, 
las opiniones y valoración de los prosumidores dentro del mundo online conducen a experiencias emocionales offline, reactivando la necesaria relación entre las dos esferas.

La participación de los prosumidores en la difusión de contenidos, tanto en intensidad como en alcance -fruto de la viralidad-, logra una repercusión más allá de los círculos y las redes más próximas. El formato de esta comunicación es narrativo en la mayoría de los casos, dentro de la estrategia de storytelling (Salmon, 2008) con la que las organizaciones afrontan en un escenario líquido de comunicación relatos que fraguan la imagen corporativa. Al storytelling empresarial se suman las narrativas creativas y autónomas de los prosumidores, en un sistema abierto de democratización de la experiencia que salta las barreras entre medios hasta consolidar la estrategia cross media, donde el mensaje circula por diferentes medios y formatos (Lacalle; SánchezNavarro, 2012, p. 2). Cuando los prosumidores tienen un gran poder, cuestionan la autoridad y buscan nuevas formas de relación, las organizaciones se enfrentan a la dificultad de cumplir con una estrategia previa de comunicación y, a menudo, con la resistencia por parte de los profesionales del sector e incluso con la falta de formación en estrategias digitales para gestionar la reputación online.

\section{Los usuarios terminan tejiendo una red, cuya acción colectiva y opiniones son la expresión de su poder en el mercado y, por tanto, de su impacto económico}

La escucha se ha venido definiendo como un proceso fundamental de las relaciones públicas, y lo es más aún en el actual contexto. La autonomía e independencia de las recomendaciones obliga a las empresas a un ejercicio permanente de monitorización y escucha, a partir del cual se puede establecer un diálogo, dentro de la desintermediación digital. Como venimos defendiendo, los prosumidores forman parte de los stakeholders de las organizaciones y su intervención es crucial en la generación del valor añadido.

La estrategia de la comunicación online analizada desde la escucha se basa en tres grados de proximidad y de conversación con los públicos:

- el primero, que se podría calificar de pasivo, en el que la información canaliza los intercambios y que considera a los públicos sólo en sus prácticas de recopilación de la información;

- el segundo, desde una mayor implicación en la escucha planteada, ofreciendo respuestas de la organización a los comentarios y valoraciones de los públicos;

- en el último, además de la escucha y la respuesta, la organización promueve y dinamiza los comentarios y la comunicación con los públicos, estableciendo un diálogo como una posición de alto compromiso.

Por tanto, se trata del ámbito donde se construye la reputación 'desde abajo' en confluencia entre las prácticas online y offline, estableciendo vínculos de confianza.

\section{Valores de la recomendación entre iguales}

El modelo de creciente influencia social de la recomendación entre iguales presenta muchas dificultades para la gestión desde la estrategia corporativa. La toma de conciencia del poder de los prosumidores ha conducido en la mayoría de los casos a una gestión orientada a los riesgos para la reputación corporativa, pero a menudo se olvida el objetivo fundamental de consolidar las relaciones con los diferentes grupos de interés, que sería el objetivo de las relaciones públicas. Aceptar las prácticas sociales, el acceso a los contenidos a través de la web social, el diálogo horizontal e igualitario, así como la atención a las demandas de transparencia, al compromiso del equilibrio entre intereses económicos y sociales son aspectos esenciales de la reputación online.

El estudio de las prácticas de los usuarios es básico para comprender las claves de la comunicación distribuida en el contexto digital, pero también para valorar su impacto y participar en la conversación dentro de la lógica de la influencia de las relaciones públicas. Así, para el análisis del impacto de la recomendación entre iguales en la reputación online hay que estimar estos factores:

- La actividad y comunicación de los prosumidores debe ser el centro del análisis. Es más, en sí mismo, el prosumidor es una categoría de análisis por cuanto, como venimos defendiendo, es a la vez canal y mensaje de la comunicación corporativa en el modelo descentralizado de la red.

- El valor de la conversación, de la interactividad, de la autonomía y de la transparencia de las prácticas de los prosumidores, en un contexto social en el que las empresas deben formar parte de la conversación.

- La comparación de los diferentes prescriptores para determinar el papel de los influenciadores en la creación de esta reputación por el valor y el alcance de su recomendación.

- La importancia de la recomendación entre iguales en los procesos de toma de decisiones en contextos complejos, como el de la comunicación multilateral y no organizada de la red.

- El análisis de los contenidos, especialmente de los producidos por los públicos, en la toma de decisiones, especialmente para el caso en que estas propuestas de los usuarios muestren elementos negativos sobre la reputación corporativa.

- La comparación de los efectos en la reputación corporativa de la recomendación entre iguales con el análisis tradicional de los medios de comunicación. A pesar de su importancia, en la gestión de la comunicación se viene realizando un seguimiento exhaustivo de los medios en lugar del estudio de los comportamientos de los prosumidores.

En conjunto, las prácticas de los usuarios en la Red se pueden seguir a través de mecanismos de evaluación de los prescriptores, de su reputación y de la producción de confianza. En sí mismo, se convierte en un modelo de negocio sólido en internet, basado en las recomendaciones, porque 
explota la suma de las prescripciones individuales y el valor de su confianza con sistemas de extracción de datos o web mining -incluso en tiempo real- para producir credibilidad y orientar decisiones de compra o elecciones de servicios.

Desde el management de la comunicación corporativa con frecuencia se analizan datos de corte cuantitativo a pesar de que no reflejan prácticas complejas de relaciones, de intereses y de valores que sintetizan la interactividad los usuarios. Aunque las aplicaciones de monitorización (que pueden ayudar al estudio de los comportamientos) más habituales muestran resultados inmediatos, no son suficientes para un estudio significativo; se hace imprescindible un giro en el estudio de la comunicación online que prime la información cualitativa, por cuanto ésta es mucho más explicativa sobre elementos esenciales de la actividad de los públicos, al contribuir a desvelar las emociones, motivaciones, expectativas y experiencias en su relación con las corporaciones.

\section{La autonomía e independencia de las re- comendaciones obliga a las empresas a un ejercicio permanente de monitoriza- ción y escucha, a partir del cual se puede establecer un diálogo}

\section{Análisis de la recomendación entre iguales para la reputación corporativa}

A la hora de analizar la reputación online hay que diferenciar entre la monitorización, y la investigación y análisis de la información obtenida mediante web mining. En conjunto, el proceso de extracción de información se realiza a través de las palabras clave con las que se filtran los contenidos en todo tipo de formatos para recopilar los comentarios de los usuarios. El proceso inicial de monitorización detecta estas keywords con el filtrado de corte sintáctico, que no es suficiente para entender las opiniones y la valoración sobre los productos y servicios o los procesos de influencia que se registran sobre la imagen corporativa. Para el análisis y la investigación sería necesario que las referencias tuvieran valor semántico y, por tanto, permitieran el análisis cualitativo.

Cada una de estas estrategias responde a los desarrollos de la web y a su evolución. En primer lugar, la extracción de información a través de algoritmos de búsqueda, propia de la web 1.0, que condujo a estrategias SEO de posicionamiento que influyen en la notoriedad pero no en la reputación online. El uso social de internet también ha modificado las fórmulas de estos algoritmos, al incorporar a partir de 2009 las búsquedas sociales, es decir, las procedentes de redes sociales en función del perfil de usuario, las cuales, hoy por hoy, son uno de los factores de peso en el posicionamiento online.

En segundo lugar, el web mining o minería de datos, fruto de la web 2.0, de donde se obtiene información relevante para estudiar la recomendación, la agregación a grupos por gustos o aficiones, la clasificación y la puesta en valor de la información obtenida, es decir, permite obtener conocimiento colectivo.
El desarrollo de la web semántica o 3.0 se basa en estos resultados, en su capacidad descriptiva y también predictiva sobre los comportamientos individuales y colectivos. La combinación de técnicas inteligentes conduce a la minería de opiniones, al desarrollo de sistemas automáticos de procesamiento de información sobre las opiniones de usuarios expresadas en la Red.

Desde entonces, los principales buscadores como Google han modificado sus fórmulas con el objetivo de aproximarse a la búsqueda inteligente, a partir de la semántica y del procesamiento del lenguaje natural. Dada la mezcla y complejidad de la información que se puede recopilar, el reto se encuentra en lograr información significativa sobre las opiniones de los usuarios que resulte útil para el desarrollo de nuevos productos y servicios o bien para mejorar la reputación de las organizaciones. El resultado de esta evolución es un modelo más complejo de minería de opiniones de carácter cualitativo que permite conocer, además de los gustos de los consumidores, el impacto de una campaña o de la imagen de marca. Esta información es muy importante para que las organizaciones puedan reaccionar rápidamente y atajar las malas opiniones o los daños a la imagen corporativa.

La elección y la valoración colectiva y social de productos y servicios se sistematiza en algoritmos de recomendaciones, de aficiones comunes, para organizar la información o el conocimiento sobre determinadas materias, comprender la actividad y los comportamientos colectivos. Los algoritmos de recomendación se diseñan con diferentes criterios: en función del contenido, del contexto o de las prácticas sociales, a partir del análisis de la comunicación interactiva de los sujetos o bien de las etiquetas. Así se puede distinguir entre el tipo y la temática de los comentarios, el contexto de la comunicación como factor que influye en la recomendación y la influencia social en los procesos colaborativos.

De todas estas opciones cabe destacar los dos grandes modelos de análisis de los comportamientos:

- de una parte, el que se realiza sobre el contenido generado por los usuarios -comentarios, etiquetas, discusiones, interacciones sociales y recomendaciones-, y

- de otra, el estudio de las tareas colaborativas como fuente de investigación de la inteligencia colectiva -producción de contenido, desarrollo de software libre o juegos-, que puede desembocar en otra modalidad de predicción de los comportamientos de los usuarios.

En el mar de información que proporciona internet, la metodología debe estar orientada a la búsqueda de significados, que interprete la información de corte cualitativo en función de las palabras clave o, mejor, de segmentos textuales de interés para la evaluación semántica. La información obtenida puede ser codificada según los criterios de la investigación para analizar elementos que afectan a la reputación corporativa, insights de interés para la gestión de la comunicación. El análisis complejo de esta información contempla que los comentarios y valoraciones no tienen necesariamente que ser coherentes ni responder a objetivos concretos, pero sí tener en consideración el aspecto emocional y clasificar los sentimientos, e incluso incorporar técnicas de aprendizaje al propio sistema. 
La metodología debe incluir una sistematización progresiva.

- Primero, se debe definir el problema de análisis en función de los resultados previsibles y de distorsiones como el sentido del humor, la ambigüedad, las expresiones informales o la geolocalización.

- En segundo lugar, ha de plantear la extracción automática de información y de comentarios relacionados con la empresa o su actividad, así como sobre la competencia, a través de un proceso selectivo entre blog, foros, redes sociales o Twitter.

- En tercer lugar, debe realizar la clasificación de las opiniones según el análisis semántico (Balahur; Montoyo, 2009), de las emociones en las opiniones (Wiebe; Wilson; Cardie, 2005), la desambiguación (Gutiérrez et al., 2010), la informalidad de los textos (Mosquera; Moreda, 2011) y la geolocalización de las opiniones. Todo ello se presenta en un resumen eficaz para la reputación online, en función del comportamiento de los prosumidores y usuarios, y de su libre expresión de corte naturalista, que adquiere significado en su investigación.

\section{Conclusiones}

Si entendemos la figura del prosumidor como stakeholder de las organizaciones, el interés sobre sus acciones en la comunicación y su influencia en la reputación corporativa es definitivo, por cuanto se trata de un agente esencial en la propia organización. De ahí la necesidad de aplicar metodologías cualitativas al estudio de la conversación en las redes sociales, por la autonomía e independencia con la que los prosumidores afectan a la reputación corporativa. La perspectiva para este estudio debe ser un planteamiento integrador e interdisciplinar, incorporando a especialistas de diferentes perfiles para atender a la complejidad y el carácter holístico de la reputación corporativa. Nos referimos a:

- expertos en comunicación con formación tecnológica para estudio de la interactividad y de los comportamientos;

- tecnólogos que mejoren las posibilidades y las herramientas de análisis;

- perfiles analíticos y de investigación social que interpreten los grandes volúmenes de datos; y

- economistas que a partir del análisis multidisciplinar orienten a la obtención de valor y la cuantificación de resultados.

Es necesario desarrollar una nueva filosofía en la evaluación de la información obtenida y en su análisis dentro de la estrategia de la comunicación corporativa, para centrarse en el valor de las opiniones y prescripciones de los prosumidores que, de forma desinteresada, proponen en función de su experiencia para la orientación de otros consumidores.

El seguimiento y análisis de la recomendación entre iguales permite intervenir en la conversación y dar muestras del grado de apertura corporativa, de mayor adaptación al contexto digital: es una oportunidad para la gestión relacional entre la organización y los stakeholders. Además, hay que insistir en que la recomendación entre iguales aporta información esencial para lograr la mayor coherencia entre las acciones de las organizaciones y los compromisos públicos adquiridos.

\section{Bibliografía}

Alloza, Ángel (2010). “De Maquiavelo a la Neurociencia: las marcas que queremos". En: Villafañe, Justo (ed.). La comunicación empresarial y la gestión de intangibles en España y Latinoamérica. Madrid: Prentice Hall, pp. 131-171. ISBN: 9788483227121

Bonchi, Francesco (2011). "Influence propagation in social networks: A data mining perspective". IEEE intelligent informatics bulletin, December, v. 12, n. 1, pp. 8-16.

http://www.comp.hkbu.edu.hk/ iib/2011/Dec/article1/ iib_vol12no1_article1.pdf

http://dx.doi.org/10.1109/WI-IAT.2011.286

Cleminson, Richard (2012). "La emotividad del mercado". Teknokultura, v. 9, n. 1, pp. 117-121.

http://teknokultura.net/index.php/tk/article/view/32

Gutiérrez, Yoan; Fernández, Antonio; Montoyo, Andrés; Vázquez, Sonia (2010). "UMCC-DLSI: Integrative resource for disambiguation task". En: Procs of the $5^{\text {th }}$ Intl workshop on semantic evaluation, pp. 427-432. Uppsala, Sweden. ISBN: 9781932432701

http://www.aclweb.org/anthology/S10-1095

Gutiérrez-Rubí, Antoni; Freire, Juan (2013). El manifiesto Crowd. Laboratorio de tendencias. ISBN: 9788493906672 http://laboratoriodetendencias.com

Hernández, Jerusalem (2011). "Reputación online: Necesidad de un marco conceptual y modelo de gestión". En: Villafañe, Justo (ed.). La comunicación empresarial y la gestión de intangibles en España y Latinoamérica. Madrid: Prentice Hall, pp. 197-214. ISBN: 9788483227121

Himanen, Pekka (2002). La ética del hacker y el espíritu de la era de la información. Barcelona: Destino. ISBN: 9788423336371

http://eprints.rclis.org/12851/1/pekka.pdf

Lacalle, Charo; Sánchez-Navarro, Jordi (2012). “Audiovisual 2.0: narrativas, recepción y consumo en los nuevos hipertextos". Anàlisi. Quaderns de comunicació i cultura, n. extra 1, pp. 1-3. https://goo.gl/qDEE2i

Levine, Rick; Locke, Christopher; Searls, Doc; Weinberger, David (1999). The Cluetrain manifesto. The end of business as usual. New York: Cluetrain. ISBN: 9780738204314 http://www.cluetrain.com

Lévy, Pierre (2004). Inteligencia colectiva. Washington: Organización Panamericana de la Salud. ISBN: 2707126934 http://inteligenciacolectiva.bvsalud.org/public/documents/ pdf/es/inteligenciaColectiva.pdf

Light, David A. (2001). "Sure, you can trust us". Sloan management review, v. 43, n. 1.

http://sloanreview.mit.edu/article/ebusiness-sure-you-cantrust-us

McLuhan, Marshall; Nevitt, Barrington (1972). Take today: The executive as dropout. New York: Harcourt Brace Jovanovish. ISBN: 9780151878307

Mosquera, Alfonso; Moreda, Paloma (2011). "Caracterización de niveles de informalidad en textos de la web 2.0". 
Procesamiento del lenguaje natural, n. 47, pp. 171-177. Alicante: Sociedad Española para el Procesamiento del Lenguaje Natural, Universidad de Alicante.

https://rua.ua.es/dspace/bitstream/10045/18525/1/ PLN_47_18.pdf

O'Reilly, Tim (2005). What is Web 2.0. Design patterns and business models for the next generation of software.

http://www.oreilly.com/pub/a/web2/archive/what-isweb-20.html

Pérez, Rafael-Alberto; Massoni, Sandra (2009). Hacia una teoría general de la estrategia. El cambio de paradigma en el comportamiento humano, la sociedad y las instituciones. Madrid: Ariel. ISBN: 9788434413108

Salmon, Christian (2008). Storytelling. La máquina de fabricar historias y formatear las mentes. Barcelona: Ediciones Península. ISBN: 9788483078358

San-Millán-Fernández, Elvira; Medrano-García, MaríaLuisa; Cavarkapa, Branko (2009). "Identidad y reputación digital: metodología en MK.20". En: XXIII Congreso anual de la European Academy of Management and Business Economics. Sevilla: Aedem. ISBN: 9788473566094

Stebbins, Robert (1996). "Cultural tourism as serious leisure". Annals of tourism research, v. 23, n. 4, pp. 948-950. http://dx.doi.org/10.1016/0160-7383(96)00028-X

Tapscott, Don (1997). La economía digital. Colombia:
McGraw Hill; Interamericana de Colombia. ISBN: 978 9586005616

Tapscott, Don; Williams, Anthony D. (2007). Wikinomics: La nueva economía de las multitudes inteligentes. Barcelona: Paidós Ibérica. ISBN: 9788449320149

Toffler, Alvin (1981). La tercera ola. Barcelona: Plaza \& Janés. ISBN: 9788401374500

https://cruceshernandezguerra.wikispaces.com/file/view/ La+tercera+ola.pdf

Westbrook, Robert A. (1987). "Product/consumption-based affective responses and postpurchase processes". Journal of marketing research, v. 24, n. 3, pp. 258-270.

http://dx.doi.org/10.2307/3151636

http://www.jstor.org/stable/3151636

Wiebe, Janyce; Wilson, Theresa; Cardie, Claire (2005). "Annotating expressions of opinions and emotions in language". Language resources and evaluation, v. 39, n. 2-3, pp. 165-210. http://www.cs.cornell.edu/home/cardie/papers/ Ire05withappendix.pdf http://dx.doi.org/10.1007/s10579-005-7880-9

Williamson, Kirsty; Stayner, Richard (1980). "Information and library needs of the aged". The Australian library journal, v. 29, n. 4, pp. 188-195

http://www.tandfonline.com/doi/pdf/10.1080/00049670.1 980.10755425

http://dx.doi.org/10.1080/00049670.1980.10755425

\section{Actualización del IF y del SJR}

En junio de 2016 SCImago y Thomson Reuters han publicado sendas actualizaciones de los indicadores de impacto de las revistas que indexan en sus respectivas bases de datos.

\section{SJR}

0.6

0.4

0.2

0

2007

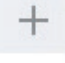

$0.422(2015)$

El Impact Factor (IF) que elabora Thomson Reuters sobre las bases de datos Science Citation Index y Social Sciences Citation Index lambas integradas en la Web of Sciencel y que presenta en la base de datos Journal Citation Reports (JCR) también muestra un buen incremento de la popularidad de esta revista El profesional de la información: hemos pasado de

$$
\text { IF } 2014=0,356 \text { a IF } 2015=0,710(+99,4 \%)
$$

El grupo de investigación español SCImago, por encargo de Elsevier, elabora el indicador SCImago Journal Rank (SJR) basado en las citas que reciben las revistas indexadas en Scopus.

Esta revista El profesional de la información ha pasado de

SJR $2014=0,374$ a SJR $2015=0,422(+12,8 \%)$

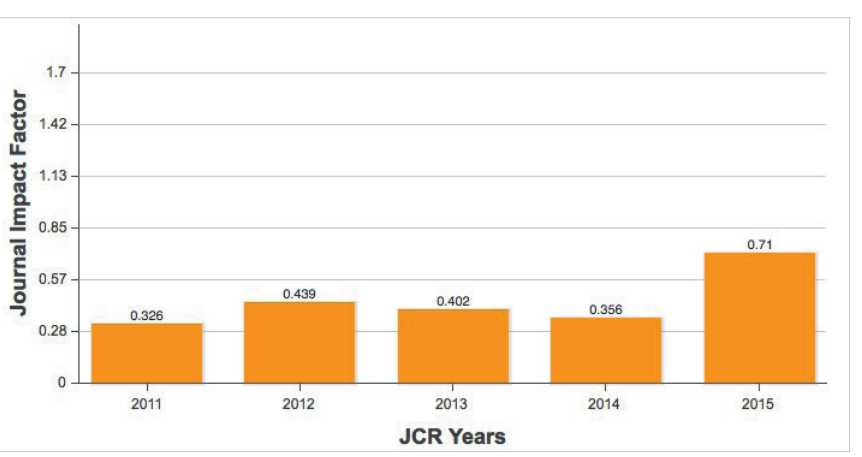

Damos las gracias a nuestros autores y a nuestros lectores 